Cahiers
Recherche
surles Droits Fondamentaux

\section{Cahiers de la recherche sur les droits} fondamentaux

17 | 2019

La motivation des actes administratifs. Le droit français à la lumière du droit administratif comparé

\title{
Le précédent juridictionnel : le pouvoir du juge dans la motivation des actes administratifs en Colombie
}

\author{
Luisa Fernanda García López
}

URL : https://journals.openedition.org/crdf/3830

DOI : $10.4000 /$ crdf.3830

ISSN : 2264-1246

Éditeur

Presses universitaires de Caen

Édition imprimée

Date de publication : 31 décembre 2019

Pagination : 171-179

ISBN : 978-2-84133-960-0

ISSN : $1634-8842$

Référence électronique

Luisa Fernanda García López, « Le précédent juridictionnel : le pouvoir du juge dans la motivation des actes administratifs en Colombie », Cahiers de la recherche sur les droits fondamentaux [En ligne], 17। 2019, mis en ligne le 06 février 2021, consulté le 15 novembre 2022. URL : http://

journals.openedition.org/crdf/3830; DOI : https://doi.org/10.4000/crdf.3830 


\title{
Le précédent juridictionnel ${ }^{1}$ : le pouvoir du juge dans la motivation des actes administratifs en Colombie
}

\author{
Luisa Fernanda GARCÍA LÓPEZ \\ Professeure principale de droit public, droit constitutionnel et droit constitutionnel comparé à l'université El Rosario de Bogota (Colombie)
}

I. La Cour constitutionnelle créatrice du précédent constitutionnel, source obligatoire de droit

A. Le précédent juridictionnel en application de la confiance légitime

B. Le précédent à partir de l'action de tutela contre "les voies de fait ", provenant d'un arrêt

II. Le Conseil d'État: un juge interprète ou créateur de droit?

L'administration entre la loi et la jurisprudence

A. Le précédent administratif: entre l'indépendance des juges et la sécurité juridique

B. L'obligation des autorités administratives de prendre en considération

les décisions du Conseil d'État et de la Cour constitutionnelle

La Cour constitutionnelle colombienne, dans son rôle d'interprète de la Constitution, a construit toute une doctrine autour de l'importance de la jurisprudence et du caractère obligatoire de celle- $\mathrm{ci}^{2}$. L'article 241 de la Constitution invoque le rôle de la Cour constitutionnelle, et une des activités essentielles de celle-ci (article 241-9) est la révision des actions de tutela. La tutela, telle qu'elle se dénomme en droit colombien, est une action en justice que toute personne peut former pour la protection des droits fondamentaux. C'est une action qui invoque la protection d'un droit fondamental lorsque celui-ci est menacé ou atteint par une autorité administrative ou judiciaire, ou même par un particulier. La tutela est une procédure d'urgence qui doit être résolue dans les dix jours suivant la présentation de l'action. Le juge doit alors définir si l'atteinte au droit fondamental est réelle et sa décision correspond à un ordre péremptoire pour protéger le droit ou faire cesser l'atteinte au droit fondamental. La tutela a pris l'ampleur d'une révolution juridique, et a connu un grand essor depuis sa création en 1991, car, malgré le fait que c'est une action subsidiaire et exceptionnelle, elle est devenue la voie «ordinaire» pour remédier à une justice assez lente et peu efficace. En effet, la tutela peut être présentée verbalement et sans représentation d'un avocat, dans le cadre d'une procédure très informelle. Elle a transformé le «scénario juridictionnel» en Colombie et, de ce fait, a permis le développement du précédent jurisprudentiel, en tant que source obligatoire de droit. En effet, le système colombien appartient à la famille du droit civil, donc marqué par une forte prédominance de la loi et du principe

1. Il faut préciser que le mot «juridictionnel» dans ce contexte veut exprimer le sens générique du précédent et sa provenance des hautes cours, ce qui suggère que le précédent judiciaire relève de la Cour suprême, la plus haute cour en matière de justice ordinaire ou judiciaire, et que le précédent juridictionnel administratif relève du Conseil d'État, la plus haute cour en matière contentieuse administrative.

2. Cet article est le produit d'une série de recherches dans le cadre du programme de recherche «Confluences entre droit public et droit privé» mené par le groupe de droit privé de l'université El Rosario de Bogota (Colombie). 
de légalité. De ce fait, la valeur de la jurisprudence avait, jusqu'en 2001, un caractère accessoire. Avant l'arrêt C-836 de 2001 qui a érigé officiellement la doctrine du précédent obligatoire, il existait une « doctrine probable» qui accordait une valeur de source à la jurisprudence, uniquement de façon exceptionnelle. Aujourd'hui, le précédent joue un rôle déterminant dans le droit colombien et le droit administratif n'est pas l'exception. Le Code administratif colombien (CPACA) ${ }^{3}$ a inclus la jurisprudence comme source obligatoire des motivations des actes administratifs et le recours extraordinaire dit d'" extension de la jurisprudence» ou extensión de jurisprudencia comme une nouveauté. C'est ainsi que la jurisprudence des hautes cours, et en particulier du Conseil d'État en tant que cour d'unification de la jurisprudence, crée un précédent dont l'administration et la justice contentieuse doivent entièrement tenir compte.

Ainsi se pose en Colombie l'important problème de la portée du précédent juridictionnel, comme source principale dans la motivation des actes administratifs. Quelles sont les implications de ces décisions? Dans quelle mesure l'administration est-elle censée se soumettre aux décisions des juges et du Conseil d'État? Comment concilier les principes de séparation des pouvoirs, sécurité juridique et confiance légitime? Le fondement de cette institution est d'abord que l'administration motive ses décisions de façon similaire au juge, que le fonctionnaire public ait l'obligation de décider en se référant à ce qui a déjà été décidé par la justice, afin d'éviter que le particulier aille systématiquement devant la justice pour obtenir une solution. Enfin, il s'agit de faire en sorte que le citoyen dispose d'une garantie d'uniformité dans les décisions, afin que sécurité juridique, bonne foi et confiance légitime soient une constante dans les décisions de l'administration et de la justice ${ }^{4}$.

Les articles 10 et 269 du CPACA consacrent le caractère obligatoire de la jurisprudence pour l'administration, ainsi que l' «extension de la jurisprudence», un recours extraordinaire qui doit garantir une interprétation uniforme de la loi. Cela signifie que l'interprétation doit se dérouler "dans les mêmes termes que la jurisprudence du Conseil d'État», en accord avec des situations de fait similaires, qui admettent un traitement uniforme par le droit, d'où l'expression «unification de jurisprudence». La nouveauté de cette institution date de 2011 et tend avant tout à ce que l'administration applique et interprète de façon uniforme la loi, dans les mêmes termes que le Conseil d'État. Il suffit que le Conseil d'État ait prononcé un jugement ${ }^{5}$ pour que cette doctrine soit la référence interprétative de l'administration et de la juridiction contentieuse administrative. Cependant, si l'administration refuse de motiver sa décision sur la base de la jurisprudence du Conseil d'État, dans les trente jours qui suivent la décision de l'administration, le plaignant peut aller devant le Conseil d'État et, dans le cadre d'une procédure extraordinaire, demander l'extension de la jurisprudence. Quels sont les enjeux de cet effort d'harmonisation entre l'administration et la justice contentieuse administrative?

Nous aborderons d'abord le contexte constitutionnel et le caractère obligatoire du précédent constitutionnel, pour lesquels la Cour constitutionnelle a elle-même établi les bases $\mathrm{du}$ " précédent juridictionnel $»^{6}(\mathrm{I})$, pour ensuite aborder «le précédent juridictionnel administratif» du Conseil d'État qui, cette fois-ci, est une création du législateur contenue dans le CPACA (II). En effet, l'uniformité, l'extension et l'unification de la jurisprudence sont des dispositions qui obligent l'administration avant tout à se soumettre au précédent établi par le Conseil d’État, comme source fondamentale du droit administratif.

\section{La Cour constitutionnelle créatrice du précédent constitutionnel, source obligatoire de droit}

La tutela est un moyen efficace pour revendiquer des droits qui auparavant n'étaient pas réellement reconnus. Grâce au développement jurisprudentiel, la Cour a accepté des droits sociaux comme ayant un contenu fondamental: la santé, la sécurité sociale, le droit au travail. Elle est toutefois devenue une Cour trop entreprenante dans l'exécution de ses compétences: par le biais de la tutela elle a donné lieu au précédent obligatoire en application du droit fondamental à l'égalité. En effet, si en principe les effets des décisions issues de l'action de tutela étaient inter-partes, la Cour a étendu le principe intercom unis si bien que toute action de tutela qui protège une situation semblable à celle qui a déjà été jugée peut être invoquée par le biais du droit fondamental à l'égalité. De ce fait, le précédent jurisprudentiel est devenu une source obligatoire pour le juge, ce qui change d'emblée le scénario juridique colombien.

La Cour constitutionnelle a développé une doctrine à propos du rôle de la jurisprudence et de son devoir de "préserver la sécurité juridique» afin qu'il y ait une certaine prévision dans les décisions, sans oublier le principe d'indépendance des juges. La sécurité juridique renvoie à d'autres principes comme la bonne foi, une évolution de la confiance légitime (A). Le précédent a trouvé une issue favorable lorsque la Cour constitutionnelle a autorisé l'action de tutela contre des arrêts provenant de la justice judiciaire ou contentieuse administrative (B).

3. Código de Procedimiento Administrativo y de lo Contencioso Administrativo, loi 1437 de 2011.

4. D. Rojas Betancourth, « $;$ En qué va la extensión de las sentencias de unificación de la jurisprudencia ?», in Instituciones de derecho administrativo, H. A. Arenas Mendoza (dir.), Bogota, Ibáñez - Universidad del Rosario, 2016, t. II, p. 507-556.

5. Ce jugement doit avoir reconnu un droit sur la base d'une loi. Pour que ce jugement devienne une référence en la matière, donc un précédent, l'administration doit vérifier si les mêmes faits de la décision s'appliquent à la situation en cours. Si tel est le cas, elle doit émettre une décision fondée sur la même loi et sur la même jurisprudence retenus par le Conseil d'État. Ainsi se construit la doctrine de l'unification de la jurisprudence et du caractère obligatoire des décisions du Conseil d'État.

6. Traduction plus proche de l'expression espagnole «precedente judicial». Toutes les traductions de l'espagnol sont les nôtres. 


\section{A. Le précédent juridictionnel en application de la confiance légitime}

La notion de précédent juridictionnel trouve sa justification dans l'interprétation faite par les hautes cours, dans le respect des principes comme l'égalité et la sécurité juridique. De ce fait, le précédent oblige toutes les autorités administratives et juridictionnelles de l'ordre national, régional ou local. En somme, toutes les autorités doivent respecter les précédents juridictionnels des hautes cours, car celles-ci sont chargées d'interpréter et de définir le contenu des lois (ordres judiciaire et administratif) et la Constitution (ordre constitutionnel). C'est ce que la Cour constitutionnelle dénomme une conception élargie du principe de légalité, lorsqu'elle interprète l'article 230 de la Constitution?. Elle affirme que les fonctionnaires sont soumis aux règles de droit positif mais aussi aux interprétations provenant des hauts magistrats. La Cour construit la doctrine du précédent, et exige une solide argumentation du juge inférieur écartant ses décisions.

L'article 230 de la Constitution politique colombienne signale que les juges dans leurs décisions sont soumis à la loi. La décision C-836 de 2001 se penche sur la valeur et la portée de la jurisprudence ${ }^{8}$ et les conditions dans lesquelles les juges inférieurs à la Cour suprême peuvent ne pas appliquer les décisions de cette dernière alors même que la jurisprudence de cassation joue un rôle d'unification et d'uniformité de la jurisprudence.

La Cour constitutionnelle dans cet arrêt a développé toute une doctrine à propos du rôle de la jurisprudence et de son devoir de "préserver la sécurité juridique» afin qu'il y ait une certaine prévision dans les décisions, ceci sans oublier le principe d'indépendance des juges. En effet, la sécurité juridique et la bonne foi ${ }^{9}$ marquent une évolution de la confiance légitime, en tant que garantie des décisions prévisibles en adéquation avec la loi. La Cour explique que l'accès à l'administration de la justice emporte un principe de confiance légitime dans l'action de l'État, la confiance légitime n'étant pas uniquement garantie par la publicité de la loi et le principe de légalité, mais par une interprétation raisonnable, uniforme et consistante de la part des juges ${ }^{10}$. Elle définit le principe de confiance légitime

[...] comme une situation où l'administré ne possède pas un droit acquis, mais peut avoir une certaine confiance dans les décisions de l'administration qui le protégeront dans sa situation, l'administration n'opérant pas de changements imprévisibles ${ }^{11}$.

De ce fait, la Cour constitutionnelle conclut que les décisions de la Cour suprême sont obligatoires pour les juges inférieurs, puisque la justice doit être conçue comme un ensemble dont l'organisation répond à un principe de hiérarchie. De ce dernier, il découle que les interprétations faites par la Cour suprême sont obligatoires pour les juges du même ordre de juridiction. La Cour suprême en tant que tribunal de cassation construit des principes qui sont bâtis comme des règles et des sources qui intègrent le système juridique. La Cour conclut que la fonction du juge obéit à un objectif de transformation sociale, car la création du droit par les juges doit s'adapter aux réalités et aux besoins sociaux qui sont en évolution permanente. La doctrine du précédent, affirme la Cour, ne peut pas être rigide au point de sacrifier des principes et des valeurs sociales qui évoluent avec le temps. De ce fait, la jurisprudence unifie l'ordre juridique.

Elle accepte les revirements jurisprudentiels pour des raisons assez explicites ${ }^{12}$, tel le renvoi au principe d'égalité, lorsque le juge donne application au principe d'égalité devant la loi et oblige, en quelque sorte, les juges à donner suite à ses interprétations. L'égalité dans l'application de la loi par les juges est en relation avec le droit fondamental à l'égalité (article 13 de la Constitution) qui se traduit par un traitement semblable de la part des autorités. En ce sens, le juge ne doit pas juger de façon différente deux situations similaires sauf si le traitement différent est justifié par des arguments raisonnables. Pour expliquer comment atteindre l'unité dans l'ordre juridique, la Cour utilise l'argumentation de l'unification jurisprudentielle. Laisser chaque juge accorder un sens différent à une même loi lorsqu'il argumente ses décisions engendrerait une incertitude juridique.

Il est donc indispensable d'établir des moyens qui donnent une uniformité à la jurisprudence, c'est ce qui est soutenu par la Cour constitutionnelle en ce qui concerne l'action de tutela.

\section{B. Le précédent à partir de l'action de tutela contre "les voies de fait", provenant d'un arrêt}

Le précédent a trouvé une issue favorable lorsque la Cour constitutionnelle a autorisé l'action de tutela contre des arrêts prononcés dans le cadre de la justice judiciaire ou administrative. En effet, suite à cette jurisprudence de la Cour constitutionnelle, une tutela peut, en dix jours, changer l'issue d'un procès ordinaire ou administratif qui aura duré entre deux ou trois ans. En effet, l'arrêt C-590 de 2005 a développé un critère permettant la présentation

7. Article 230 de la Constitution colombienne: «Les juges dans leurs décisions sont soumis uniquement à la loi. L'équité, la jurisprudence, les principes généraux du droit ainsi que la doctrine sont des critères auxiliaires de la fonction judiciaire juridictionnelle».

8. La jurisprudence de la Cour suprême de justice pour les juges inférieurs, le précédent vertical et l'avenir des futures décisions de la Cour suprême et le précédent horizontal.

9. Article 83 de la Constitution colombienne.

10. Cour constitutionnelle colombienne, arrêt C-836 de 2011, magistrat rapporteur: Rodrigo Escobar Gil.

11. Cour constitutionnelle colombienne, arrêt C-478, 1998, magistrat rapporteur: Alejandro Martinez.

12. Cour constitutionnelle colombienne, arrêt T-256, 1993, magistrat rapporteur: Eduardo Cifuentes Muñoz. 
des actions de tutela contre des arrêts dont la procédure ou la décision constituent des voies de fait.

La jurisprudence de la Cour constitutionnelle a créé un précédent concernant la possibilité de présenter des actions de tutela contre des arrêts. De ce fait, elle a permis d'invoquer la tutela en cas d'atteinte contre un droit fondamental commise par un juge ou au cours d'une procédure devant un juge. La Cour a initialement prévu plusieurs conditions pour invoquer la tutela contre arrêt:

- que l'arrêt porte atteinte à un droit fondamental;

- que le plaignant ait épuisé toutes les voies de recours ordinaires et extraordinaires prévues par la justice avant d'invoquer l'action de tutela;

- que les circonstances montrent un caractère immédiat pour justifier la présentation de la tutela; ceci en accord avec le caractère raisonnable et la proportionnalité;

- qu'il s'agisse d'un défaut de procédure qui porte atteinte aux droits fondamentaux;

- que le plaignant invoque les fautes commises par le juge dans le procès en cause et que le juge n'en tienne pas compte;

- que l'arrêt qui comporte une atteinte grave à un droit fondamental ne soit pas la suite d'une action de tutela.

Par la suite, dans la même décision ${ }^{13}$, la Cour a précisé qu'une des conditions doit être remplie pour qu'il y ait une atteinte directe à la Constitution. Ce sont des voies de fait. La tutela est alors invocable:

- lorsque le juge justifie sa décision sur la base d'une loi ou d'une disposition inexistante, ou que la décision repose sur une disposition abrogée et n'a aucun fondement juridique;

- de même, lorsque la norme qui justifie la décision demande des interprétations accessoires d'autres normes dont il n'a pas été tenu compte pour le cas concret;

- lorsque l'application de certaines normes constitutionnelles porte atteinte aux droits fondamentaux dans le cas concret;

- lorsque la décision n'est pas en accord avec les arguments juridiques de la décision;

- lorsque les motivations juridiques ne sont pas en adéquation avec la décision;
- lorsqu'une disposition de droit positif méconnaît les effets d'un arrêt erga omnes, et va à l'encontre de sa ratio decidendi.

A priori, la tutela contre arrêt est exceptionnelle. En réalité, c'est une pratique qui devient de plus en plus courante lorsque les interprétations des juges sont en désaccord avec celles de la Cour constitutionnelle. En effet, la Cour signale, dans son arrêt C-067 de 2012, que l'herméneutique légale est celle qui oriente les interprétations de la Cour: la méthode de «l'interprétation conforme» à la Constitution doit toujours prévaloir sur toute autre interprétation. C'est le cas de la déclaration de caducité de l'action en réparation directe, et de la soumission au précédent de la Cour constitutionnelle.

Les exemples sont nombreux, mais un cas est récurrent, lorsque le juge administratif déclare la caducité de l'action en réparation en cas de responsabilité de l'État. En effet, l'action en réparation directe est un recours en indemnisation pour des dommages causés par l'action ou l'omission des autorités publiques ${ }^{14}$. Le délai de caducité de deux ans court à partir du moment où l'action ou l'omission ont causé le dommage. S’il s'agit du délit de disparition forcée, le délai court à compter de la date où la victime est libérée mais la demande peut être présentée à partir du moment de la disparition de la victime. L'arrêt T-156 de 2009 montre le cas d'une action de tutela présentée par une mère ayant formé une action en réparation directe. Elle a demandé à un hôpital une indemnisation pour faute médicale. Le tribunal administratif du département de Bolivar a décidé que l'action était caduque, bien que le Conseil d'État ait auparavant affirmé que, lorsqu'il y a des incertitudes, s'il existe vraiment une responsabilité de l'État, il n'est pas nécessaire de définir les dates exactes pour demander la réparation. La Cour qui a examiné cette situation par une tutela a estimé que l'absence de définition de date n'était pas une condition suffisante pour déclarer la caducité de l'action. Dans ce sens, l'arrêt du tribunal administratif de Bolivar démontre un défaut substantiel ${ }^{15}$ et de fait ${ }^{16}$, ce qui justifie l'action de tutela contre cet arrêt ${ }^{17}$.

L'arrêt T-075 de 2014 évoque également une action de tutela contre arrêt. Dans une affaire de responsabilité médicale devant le tribunal administratif de Tolima, la Cour décide que, même s'il n'est pas possible de définir les dates auxquelles le dommage a été causé par les responsables d'un service, la caducité de l'action en réparation

13. Cour constitutionnelle colombienne, arrêt C-590, 2005, magistrat rapporteur: Jaime Córdoba Triviño.

14. Article 140 CPACA, loi 1497 de 2011: "Aux termes de l'article 90 de la Constitution, le plaignant peut demander directement la réparation des dommages antijuridiques» causés par l'acte ou l'omission des agents de l'État. Le dommage antijuridique est la base de la responsabilité patrimoniale de l'État, consacrée dans l'article 90 de la Constitution. C'est une responsabilité objective, car la faute n'est pas une condition pour déclarer la responsabilité. Le dommage antijuridique évoque un dommage que la victime n'a pas le devoir de supporter car il constitue une charge injuste pour celle-ci. Conformément à l'alinéa précédemment cité, «l'État va réparer lorsque la cause du dommage est un fait, une omission, un fonctionnement administratif ou une occupation temporaire ou permanente de la propriété immobilière en raison de travaux publics ou pour toutes autres raisons imputables à une entité publique ou un particulier qui a agi suite à une instruction expresse [...]».

15. Défaut substantiel: lorsque le juge en exercice de son autonomie et de son indépendance va au-delà de la loi ou de la Constitution.

16. Défaut de fait: lorsque le juge ne fournit pas des preuves ou ne fait aucune analyse des preuves. Voir en ce sens les arrêts C-59o de 2005 , T-010 de 2012, SU-159 de 2002, T-550 de 2002, T-923 de 2004, T-104 de 2007.

17. Cour constitutionnelle colombienne, arrêt de tutela T-156, 2009. 
directe ne peut pas servir d'argument pour rejeter les prétentions du plaignant. Dans cet arrêt, la Cour constitutionnelle invite à suivre le Conseil d'État ${ }^{18}$ qui, dans les cas de responsabilité médicale, prévoit que le délai de l'action en réparation pour faute médicale ne peut pas débuter avec le diagnostic de la maladie. En effet, le Conseil d'État considère que l'action en réparation commence à partir du moment où la personne est au courant du dommage qu'elle a subi, lorsqu'il y a un traitement médical continu et qui engendre un espoir de guérison. Même si le patient est au courant des dommages causés par le service médical, il faudra attendre un diagnostic définitif. Le précédent du Conseil d'État dans les cas de réparation directe pour défaut dans la prestation du service médical incorpore le principe pro damnato ${ }^{19}$ : il est raisonnable de considérer que, dans certains cas, le dommage se manifeste après la survenance du fait ou l'omission de l'administration ou qu'il se prolonge dans le temps, jusqu'à ce qu'il soit accordé un diagnostic définitif et que les dommages deviennent évidents. Dans ce $\operatorname{cas}^{20}$, la tutela contre arrêt est acceptée puisque la décision du tribunal administratif de Tolima a montré un défaut substantiel ${ }^{21}$, lorsqu'il a déclaré la caducité de l'action de réparation directe pour faute de service alors que le plaignant argumentait un délai non raisonnable dans l'administration du médicament. En ce sens, la Cour a accordé la protection du droit fondamental au procès équitable et a laissé sans effet le jugement rendu par le tribunal administratif de Tolima. Par la suite, elle a ordonné au tribunal administratif de Tolima de se prononcer sur le fond du procès de la responsabilité et de la réparation directe dans un délai de vingt jours à compter de la notification de la décision.

Certes, la Cour montre bien un grand pouvoir à l'égard des autres juridictions et du législateur. À travers sa jurisprudence elle a donné un pouvoir obligatoire à ses décisions, instaurant ainsi la doctrine du précédent obligatoire. Le précédent juridictionnel constitutionnel est bien une source de droit. Néanmoins, bien qu'initialement la Cour constitutionnelle ait rendu le précédent obligatoire pour les juges, elle a par la suite considéré essentiel que l'administration soit soumise audit précédent dans la mesure où elle n'a pas la même autonomie que les juges. Dans ce sens, l'arrêt C-539 de 2011 a précisé que tous les fonctionnaires publics doivent se soumettre au précédent juridictionnel constitutionnel, c'est-à-dire à l'application et interprétation des normes, ainsi qu'à l'interprétation du juge susceptible de s'adapter à toute situation dont les faits sont similaires. Or, le juge ordinaire ou administratif doit suivre la jurisprudence constitutionnelle ${ }^{22}$ et, s'il ne le fait pas, il doit se justifier. En effet, la Constitution étant une norme au-dessus de toute autre norme ${ }^{23}$, le juge à l'obligation de suivre le précédent constitutionnel car celui-ci est une source obligatoire de droit ${ }^{24}$.

\section{Le Conseil d'État: un juge interprète ou créateur de droit ${ }^{25}$ ? L'administration entre la loi et la jurisprudence}

La pratique colombienne montre bien une confiance à l'excès envers le juge, et une certaine méfiance envers l'administration. Bien que la loi prévoie que la fonction administrative doive être soumise à la loi, aux droits de l'homme et au principe démocratique, l'administration manque de force dans l'exécution de ses actes administratifs (A). En accord avec la Constitution, l'administration a une finalité: satisfaire l'intérêt général ${ }^{26}$. En ce sens, l'État social de droit, les principes de la fonction administrative mettent en évidence que l'action de l'administration doit être orientée vers la protection de l'individu en accord avec le procès équitable, le principe de légalité, la présomption d'innocence, la non reformatio in pejus et le principe non bis in idem ${ }^{27}$. De même, l'égalité et l'impartialité sont des principes qui doivent guider l'administration ${ }^{28}$. Cependant,

18. Conseil d'État colombien, chambre contentieuse administrative, section 3, arrêt du 16 août 2001, n 18.273 , magistrat rapporteur: Alier Eduardo Hernández Enríquez; Conseil d'État colombien, chambre contentieuse administrative, arrêt du 16 août 2001, n 13.772 (1048), mentionné dans l'arrêt du 13 février 2003, nº 13.237 (Rad. 2555), magistrat rapporteur: Ricardo Hoyos Duque.

19. Principe qui implique une certaine bienveillance pour les victimes et titulaires du droit à la réparation. Conseil d'État colombien, section 3 , arrêt du 10 avril 1997, $\mathrm{n}^{\circ}$ 11.954, magistrat rapporteur: Ricardo Hoyos Duque, et communication du 7 mars 2002, $\mathrm{n}^{\circ}$ 21.189, conseiller rapporteur: Ricardo Hoyos Duque (référence citée dans l'arrêt du Conseil d'État colombien, section 3, Rad. 27141, 20 février 2014, magistrat rapporteur: Danilo Rojas Betancourth).

20. Cour constitutionnelle colombienne, arrêt de tutela T-075, 2014.

21. Un défaut d'appréciation.

22. À tel point que la Cour constitutionnelle indique que le recours en cassation (article 365 du Code de procédure civile) doit être fondé sur le fait d'ignorer la chose jugée ainsi que sur la doctrine constitutionnelle.

23. Article 4 de la Constitution colombienne.

24. Cour constitutionnelle colombienne, arrêt C-104, 1993. Dans ce sens, l'arrêt C-634, 2011 établit un paramètre supplémentaire : le caractère obligatoire du précédent constitutionnel.

25. F. Mantilla Espinosa, «Interpretar: ¿ aplicar o crear derecho? Análisis desde la perspectiva del derecho privado », Revista de derecho de la Pontificia Universidad Católica de Valparaíso, vol. 33, n² 2, 2009, p. 537-597.

26. G. Sánchez Luque, «El Código de Procedimiento Administrativo y de lo Contencioso Administrativo: hacia la efectividad de los derechos », in Instituciones de derecho administrativo, p. 557-586.

27. Article 29 de la Constitution colombienne: «Les règles du procès équitable doivent être respectées dans l'élaboration de tous les actes juridictionnels et administratifs. Toute personne sera jugée conformément aux lois en vigueur devant un juge ou tribunal compétent et dans le respect absolu des formes établies pour chaque procédure».

28. Article 209 de la Constitution colombienne: «La fonction administrative est au service de l'intérêt général en conformité avec les principes d'égalité, de moralité, d'efficacité, d'économie, de célérité, d'impartialité, et de publicité et s'exerce à travers la décentralisation, la délégation et la déconcentration des fonctions". 
la notion de précédent dans la jurisprudence du Conseil d'État est récente. En effet, suite au développement jurisprudentiel de la Cour constitutionnelle et la réforme du Code du contentieux administratif, le précédent s'impose dans le domaine administratif.

Le caractère obligatoire du précédent en droit administratif date de la loi 1437 de 2011 (CPACA) dont les articles 10, 102 et 269 ont incorporé le mécanisme d'extension de jurisprudence en tant que mesure visant à réduire l'encombrement de la juridiction administrative (B).

La portée du précédent juridictionnel dans le droit administratif ${ }^{29}$ répond à une idée fondamentale: les autorités administratives doivent appliquer les dispositions constitutionnelles, légales et réglementaires de façon uniforme et en accord avec les interprétations du Conseil d'État, dans toute situation présentant une identité en termes de fait et de droit.

L'article 102 crée l'extension de la jurisprudence du Conseil d'État à des tiers par les autorités administratives: «Les autorités doivent étendre les effets de tout arrêt d'unification de jurisprudence du conseil d'État constitutive d'une reconnaissance d'un droit [...]». Cette procédure administrative s'applique lorsque le particulier demande à l'autorité administrative l'extension des effets d'un arrêt d'unification reconnaissant un droit. Cette demande doit se faire dans un laps de temps de trente jours. Cette requête exige que le particulier se retrouve dans la même situation de fait et de droit que celle concernée par l'arrêt d'unification et que l'action juridictionnelle permette de réclamer la protection du droit. Lorsque le particulier conteste une réponse de l'administration, il peut saisir le juge administratif (article 269 CPACA), lequel doit adopter la jurisprudence du Conseil d'État. L'arrêt SU-611 de 2017 confirme que le Conseil d'État, à son tour, doit suivre la jurisprudence de la Cour constitutionnelle ainsi que sa propre jurisprudence.

\section{A. Le précédent administratif: entre l'indépendance des juges et la sécurité juridique}

Au-delà des discussions théoriques relatives au changement de structure des sources du droit ${ }^{30}$, la notion de précédent présente un contenu pratique pour le droit administratif. En effet, les décisions de l'administration qui sont soumises au contrôle juridictionnel permettent de définir la portée de la décision administrative et de déterminer si elles sont en accord avec les interprétations du Conseil d'État. Si une décision ne suit pas le précédent et ne justifie pas les raisons pour lesquelles elle s'écarte de celui-ci, la décision de l'administration n'aura aucune validité, ce qui correspond à un acte administratif nul. Cela peut même entraîner la responsabilité de l'État si éventuellement l'acte administratif cause un dommage. D'où l'importance de la prise en compte de la jurisprudence du Conseil d'État dans le cadre de la motivation des actes administratifs. Cette prise en compte contribue à une harmonisation entre les décisions de l'administration et celles de la juridiction administrative. Les implications sont assez significatives du point de vue de la sécurité juridique puisque le Conseil d'État établit un lien entre la fonction administrative et la fonction juridictionnelle. Elles le sont également du point de vue de la décongestion des tribunaux, puisque l'alignement des décisions administratives sur celles de la plus haute autorité en matière de contentieux administratif réduit le poids du contentieux ${ }^{31}$.

En réalité cette interprétation vient de la doctrine et part de l'idée initiale des rédacteurs du CPACA. En effet, le comité rédacteur a voulu donner à l'administration les instruments pour qu'elle puisse de son propre chef motiver ses actes en suivant le précédent établi par le Conseil d'État. Cependant, l'administration n'arrive pas à s'approprier le précédent en tant que tel, et elle reste assez discrète dans la motivation de ses actes administratifs. Dans beaucoup de situations, elle préfère ne pas adopter le précédent pour deux raisons principales. D'une part, parce que le droit administratif colombien est fondé sur l'autorité de la loi et du principe de légalité. De ce fait, le fonctionnaire administratif se retrouve face à un dilemme: «la loi ou la jurisprudence?». Ceci montre bien que notre système, construit autour de la loi, est en mutation vers un système mixte, qui accorde une prépondérance à la jurisprudence comme source obligatoire de droit. Cette ambivalence nous renvoie à une deuxième problématique: une sorte d'autorité supérieure est reconnue au juge, lorsque l'administration ne motive pas ses décisions sur la base d'arrêts.

La règle du précédent répond à une volonté de donner une plus forte autorité au juge, et renvoie au principe de légalité consacré par le droit colombien. Cependant, le Conseil d'État est assez clair sur le fait que, lorsque les agents publics ne respectent pas le précédent, ils transgressent les principes d'économie, d'efficacité, d'égalité et de responsabilité administrative. De plus, l'obligation des agents publics de rendre efficaces les droits des administrés est une obligation consacrée dans le CPACA (article 31). Si le fonctionnaire ne respecte pas ce principe, il est passible de faute disciplinaire. En réalité, toute décision qui ne respecte pas le précédent juridictionnel administratif constitue une atteinte aux droits fondamentaux et est susceptible de faire l'objet d'une action de tutela ou d'engager la responsabilité pénale, administrative ou disciplinaire.

29. Article 10 CPACA : l'obligation d'application uniforme des normes et de la jurisprudence s'impose aux fonctionnaires publics pour résoudre les affaires relevant de leur compétence.

30. Voir en ce sens D. Rojas Betancourth, «¿En qué va la extensión de las sentencias de unificación de la jurisprudencia?» et opinion consultative CE 2177 de 2013, Conseil d'État colombien, Salle de consultation et du Service civil, conseiller rapporteur: William Zambrano Cetina, Bogota DC, 10 décembre 2013.

31. Ibid. 
Le Conseil d'État justifie sa position au regard de son rôle d'interprète authentique du CPACA : celui-ci appelle une interprétation conforme de la part de l'administration dans le cadre de la motivation ${ }^{32}$.

En outre, l'administration a l'obligation de suivre le précédent constitutionnel dans la procédure d'extension de la jurisprudence du contentieux administratif. En effet, la Cour constitutionnelle a souligné que son précédent a force obligatoire pour tous les opérateurs juridiques, y compris les juges. Il s'agit de matérialiser le respect des principes d'égalité, de la suprématie de la Charte politique, du procès équitable et de la confiance légitime, valeurs qui obligent les juges à prendre en compte les décisions de cette cour ${ }^{33}$.

En définitive, la Colombie intègre alors les courants de la théorie du droit: la jurisprudence est une source juridique formelle, puisque les lois sont dénuées de sens. Les préceptes légaux peuvent avoir plusieurs significations qui constituent des déclarations prescriptives différentes et sont le produit d'un processus d'interprétation. L'herméneutique établie par les autorités judiciaires qui ont le pouvoir d'unifier la jurisprudence et d'attribuer un sens aux normes plus élevées acquiert un caractère contraignant pour les autres opérateurs juridiques.

L'obligation faite à l'administration de reprendre les précédents dans le cadre de la motivation est fondée sur les arguments suivants:

- le langage ordinaire employé par les règles est ambigu au vu de ses significations multiples. Il est imprécis au vu de l'indétermination de ses concepts. Ces traits affectent l'interprétation et l'application du droit. Les problèmes qui en résultent ne sont résolus que par un processus herméneutique à travers lequel les juges créent des règles individuelles en tant qu'interprètes du système juridique. Ces règles lient les autres autorités publiques;

- les arrêts ont pour fonction d'harmoniser les lois et leurs effets juridiques défavorables;

- la jurisprudence est garante des principes fondamentaux de l'État constitutionnel, tels que celui la sécurité juridique.

\section{B. L'obligation des autorités administratives de prendre en considération les décisions du Conseil d'État et de la Cour constitutionnelle}

Les autorités administratives ne peuvent décider en toute liberté de prendre en compte les décisions du Conseil d'État. En effet, les articles 102 et 269 du CPACA relatifs à l'extension de jurisprudence, et l'article 256 du CPACA relatif au recours extraordinaire d'unification, prévoient que ce sont les décisions du Conseil d'État qui ont une valeur de précédent juridictionnel administratif.

Lorsque les autorités administratives répondent à une situation particulière par un acte administratif, elles sont donc obligées ${ }^{34}$ :

- d'identifier et de donner application aux normes qui répondent au cas concret;

- d'identifier et d'appliquer les arrêts du Conseil d'État justifiant leurs décisions. Les arrêts peuvent ou ne pas être des arrêts d'unification, et si l'autorité administrative décide de ne pas invoquer un arrêt donné, elle est obligée d'invoquer des arguments d'ordre constitutionnel pour s'écarter du précédent;

- d'accorder une importance majeure aux valeurs, principes et droits constitutionnels, en cas d'absence de précédent sur les dispositions applicables au cas concret, ou dans l'hypothèse d'interprétations contradictoires. Ainsi, il faudra argumenter plus aisément s'il existe un précédent, et préciser les raisons pour lesquelles le fonctionnaire ne l'adopte pas, s'il existe;

- enfin, de remplir toutes les conditions précédentes s'il existe un arrêt d'unification.

Quel est le rôle du Conseil d'État dans l'unification de la jurisprudence en tant que tribunal suprême ${ }^{35}$ ?

La loi 1437 de 2011 a donné une valeur supérieure à la jurisprudence du Conseil d'État à travers les arrêts d'unification. Lorsque l'administration prend des décisions, conformément à l'article 1o du CPACA, elle doit garantir l'interprétation uniforme des normes constitutionnelles et légales. L'extension de jurisprudence permet de reconnaître des droits dans le sens où des situations semblables doivent recevoir les mêmes solutions juridiques. C'est ce que le Code de procédure administrative consacre à propos des arrêts d'unification (article 102). Si l'administration ne répond pas ou ne reconnaît pas le droit en question, le particulier peut saisir la juridiction administrative afin d'obtenir l'extension de jurisprudence (article 269).

De ce fait, les arrêts d'unification produisent des effets à l'intérieur de la juridiction administrative, puisqu'elle active le recours extraordinaire d'unification de jurisprudence. L'administration dans ce sens est obligée de tenir compte des arrêts pour décider des cas similaires et d'étendre leurs effets aux citoyens qui se trouvent dans les mêmes situations. Les arrêts d'unification de jurisprudence sont les décisions du Conseil d'État qui ont une importance juridique, économique ou sociale. Ils sont

32. Opinion consultative CE 2177 de 2013, Conseil d'État colombien, Salle de consultation et du Service civil, conseiller rapporteur: William Zambrano Cetina, Bogota DC, 10 décembre 2013.

33. Cour constitutionnelle colombienne, arrêt SU-o68, 2018, action de tutela contre l'unité administrative de gestion des retraites et des contributions fiscales (UGPP: Unidad de Gestión Pensional y Parafiscales), contre le Conseil d'État, section A, magistrat rapporteur: Alberto Rojas Rios.

34. Conseil d'État colombien, section 2 A, 29 janvier 2014, magistrat rapporteur: G. Gomez Aranguren, rad. 4305-13; Conseil d'État colombien, section 4, 15 janvier 2014, magistrat rapporteur: J. Octavio Ramirez, rad. 20093.

35. Cour constitutionnelle colombienne, arrêt C-634, 2011, magistrat rapporteur: L. E. Vargas Silva; arrêt C-816, 2011, magistrat rapporteur: M. Gonzalez; et arrêt C-588, 2012, magistrat rapporteur: M. Gonzalez. 
prononcés par la chambre plénière du Conseil d'État et les sections spécialisées dans des domaines bien précis ${ }^{36}$.

Un arrêt de principe de la Cour constitutionnelle de $2012^{37}$ porte sur l'action constitutionnelle de tutela formée par un fonctionnaire pour atteinte contre ses droits fondamentaux au procès équitable et à l'égalité devant la loi. Le requérant invoque le précédent constitutionnel de l'arrêt de tutela SU-917 de 2010, dans lequel la Cour constitutionnelle pose l'obligation de motiver toutes les décisions administratives. Tout acte administratif non motivé attaqué en justice doit être déclaré nul car il constitue une atteinte aux droits fondamentaux. Cependant, la loi prévoit que l'acte administratif de licenciement ne doit pas être motivé ${ }^{38}$. Comment résoudre cette contradiction?

La Cour constitutionnelle souligne que la motivation des actes administratifs est un sujet qui a été développé par sa propre jurisprudence ${ }^{39}$ car celui-ci porte sur le droit au procès équitable. Il est certain que le procès équitable ${ }^{40}$ est une condition pour que le droit au contradictoire et à la défense soit à la portée des particuliers face aux décisions des autorités administratives. En effet, lorsque l'acte administratif n'exprime pas les raisons qui ont motivé la décision, le particulier perd un moyen de défense face à l'administration ${ }^{41}$.

Le principe démocratique justifie aussi l'obligation de motivation des actes administratifs puisque les autorités administratives ont l'obligation de rendre des comptes concernant ses actions.

La Constitution de 1991 (article 209) consacre le principe de la publicité de la fonction administrative. Grâce à cette disposition, les particuliers sont en mesure de connaître les raisons de l'action administrative. Selon la Cour, la nécessité de motiver les actes administratifs découle des principes constitutionnels tendant à éviter l'abus de pouvoir. Si l'administration doit motiver ses actes et si les juges doivent contrôler l'adéquation de cette motivation, que reste-t-il du pouvoir discrétionnaire de l'administration? La Cour affirme que pouvoir discrétionnaire ne signifie pas pouvoir absolu. L'exercice du pouvoir discrétionnaire doit se faire en conformité avec la loi. Dans le cas concret, l'arrêt de tutela T-204 de 2012, le fonctionnaire avait un statut particulier puisque, alors même qu'il était entré dans la fonction publique par concours, le poste qu'il occupait au moment de son licenciement était provisoire. Cependant, eu égard à son statut, la loi obligeait l'administration à motiver l'acte administratif mettant fin à son contrat. En effet, seul le licenciement relatif aux emplois à discrétion est exempt de la règle de motivation des actes administratifs.

Certes, la loi 909 de $2004^{42}$ sur l'emploi public et la fonction administrative reconnaît que le licenciement de tout fonctionnaire est une attribution réglementaire qui doit se faire par un acte administratif motivé. La Cour pose le principe de l'expression de la «raison suffisante» de l'acte administratif portant licenciement d'un fonctionnaire. Ainsi se trouve exclue toute justification indéfinie, abstraite. Selon la jurisprudence de la Cour constitutionnelle, toute décision de ce genre doit être motivée de façon claire, précise et détaillée. En 2003, le Conseil d'État a unifié la jurisprudence: le licenciement à des postes provisoires ne doit pas être motivé. La décision de licenciement prise par l'administration se justifie (sans motivation) par la suppression définitive du poste, ou par des sanctions disciplinaires infligées au fonctionnaire en question, ou encore par l'existence d'une note insuffisante concernant ponctuellement l'exercice de la fonction.

Le caractère obligatoire du précédent pose ici un problème fondamental pour le fonctionnaire: faut-il suivre la jurisprudence de la Cour constitutionnelle ou suivre la jurisprudence du Conseil d'État?

Le plaignant a présenté «une action en nullité et restauration du droit ${ }^{43}$, devant la justice administrative, contre la résolution 0-1573 du 24 octobre 2001 afin d'obtenir l'annulation de l'acte administratif et la réincorporation à son poste. L'arrêt du 3 décembre 2007 a jugé en première instance ${ }^{44}$, a déclaré nulle la résolution et a ordonné la réincorporation du fonctionnaire dans son poste. Ce juge de première instance a condamné l'État (pouvoir juridictionnel) à payer les salaires non perçus par le fonctionnaire jusqu'à sa réincorporation.

En deuxième instance, le 8 juillet 2011, le tribunal administratif du département du Bolivar a décidé de révoquer l'arrêt en première instance, car, en application de la jurisprudence du Conseil d'État, il n'était pas nécessaire de motiver les actes administratifs pour les fonctionnaires ayant un statut provisoire. Il a argumenté que cela obéissait au pouvoir discrétionnaire de l'administration étant donné qu'il n'existait pas de vocation de permanence dans le poste, donc pas de stabilité dans la fonction publique.

36. L'extension des effets d'un jugement de la Cour constitutionnelle (T-025, 1994) sur le thème de la "migration interne forcée» fut invoquée devant le Conseil d'État (cinquième section), où il a décidé que les effets des décisions rendues par la Cour constitutionnelle n'étaient pas susceptibles d'être prolongés à l'aide du mécanisme d'extension de jurisprudence. D'autant plus que le Conseil d'État n'a pas un tel pouvoir, étant donné que les décisions produites du contrôle abstrait (déclarations « $\mathrm{C} »$ ) contiennent des mandats impératifs et ne sont pas invocables par l'extension de jurisprudence. S’agissant du contrôle concret (déclarations « T » et «SU»), ce sont des décisions avec des contenus entre les acteurs. Les jugements de la Cour constitutionnelle peuvent alors servir de référence mais ne peuvent être invoqués en extension de jurisprudence.

37. Cour constitutionnelle colombienne, arrêt de tutela T-204, 2012, magistrat rapporteur: J. I. Palacio.

38. Ibid.

39. Article 1 de la Constitution colombienne.

40. Article 29 de la Constitution colombienne.

41. Cour constitutionnelle colombienne, arrêt de tutela SU-917, 2010, magistrat rapporteur: J. I. Palacio.

42. L'article 43-1 de la loi 909 de 2004 prévoit que le fonctionnaire public est licencié par un acte administratif motivé lorsqu'il reçoit une évaluation négative.

43. Voir l'article 137 CPACA.

44. Premier juge administratif du circuit de Carthagène. 
Le fonctionnaire licencié, non satisfait de cette décision, a présenté une action de tutela contre la décision du tribunal administratif de Bolivar, pour atteinte contre le droit au procès équitable, le droit d'accès à la justice et la non-reconnaissance du précédent juridictionnel, établi par la Cour constitutionnelle dans les arrêts SU-917 de 2010 et l'arrêt T-289 de 2011.

La Cour constitutionnelle a conclu que le fonctionnaire devait réintégrer son poste (ou être nommé à un poste de catégorie supérieure), l'entité responsable devant lui rembourser les salaires et prestations sociales non perçus.

En définitive, l'action de tutela contre les actes administratifs se matérialise dès ${ }^{45}$ :

- que l'arrêt porte atteinte à un droit fondamental;

- que le plaignant a épuisé toutes les voies de recours ordinaires et extraordinaires avant d'invoquer l'action de tutela;

- que les circonstances montrent un caractère urgent, justifiant la présentation de la tutela afin d'éviter une atteinte à une liberté fondamentale;

- qu'il s'agit d'un défaut de procédure qui porte atteinte aux droits fondamentaux;

- que le plaignant invoque les fautes commises par le juge dans le procès en cause et que, malgré cela, le juge n'en a pas tenu compte;

- que l'arrêt qui comporte une atteinte grave à un droit fondamental n'est pas la suite d'une action de tutela.

Le rôle du précédent juridictionnel dans la motivation des actes administratifs se confirme par l'extension de jurisprudence et le pouvoir du juge. Cependant, l'action de tutela reste une institution qui positionne la Cour constitutionnelle comme une cour au-dessus de toutes les hautes cours, avec un pouvoir considérable. Dans toutes les hypothèses, la Cour aura le dernier mot, car la tutela est devenue le dernier recours, même en matière d'extension de jurisprudence. L'action de tutela a pris une grande ampleur et, de toute évidence, le recours extraordinaire d'extension de jurisprudence ne sera jamais la dernière option avant l'action de tutela contre arrêts ou actes administratifs.

Nous assistons à une révolution juridique, au cours de laquelle le développement de l'action de tutela semble difficile à contenir puisque plusieurs projets de loi visant à limiter l'ampleur de la tutela sont restés sans succès. L'idéal serait de restreindre la tutela contre arrêt, afin de donner plus de légitimité aux juridictions judiciaire et administrative et éviter que la tutela ne soit une troisième instance. Ainsi, en avril 2019, un nouveau projet de réforme de la justice a été déposé au Parlement. Il tend à apporter des solutions aux nombreux problèmes qui accablent la justice, et notamment à restreindre la portée de la tutela. Il cherche surtout à renforcer le précédent juridictionnel administratif en le rendant absolument obligatoire, et donc décourager l'utilisation de la tutela contre arrêt. Selon le projet, le juge sera l'interprète authentique de la loi et son interprétation s'imposera comme la seule interprétation obligatoire ${ }^{46}$. En conséquence, la possibilité de poursuivre la procédure avec les voies de recours à la portée du plaignant, telles que la tutela, serait écartée.

45. Cour constitutionnelle colombienne, arrêt T-076, 2018, magistrat rapporteur: L. G. Guerrero Pérez.

46. G. Vargas Lleras, «Reforma a la justicia: un primer acuerdo», El tiempo, 7 avril 2019, en ligne: https://www.eltiempo.com/opinion/columnistas/ german-vargas-lleras/reforma-de-la-justicia-un-primer-acuerdo-346616. 